\title{
Incidences and predictors of HIV positivity among infants who born from HIV positive mother who have follow up at two hospitals of southern Ethiopia, 2014
}

\author{
Tariku Tadele ${ }^{1}$, Alemu Tamiso ${ }^{2}$, Tafese Tadele ${ }^{3}$ \\ ${ }^{1}$ Department of Internal medicine, School of medicine, College of Medicine and Health Sciences, Hawassa University, Hawassa, \\ Ethiopia \\ ${ }^{2}$ Department of Public health/Unit of epidemiology and biostatistics, College of Medicine and Health Sciences, Arba Minch University, \\ Arba Minch, Ethiopia \\ ${ }^{3}$ Departments of public health, Yirgalem College of medical sciences, Southern Ethiopia regional health bureau, Ethiopia
}

\section{Email address:}

tarikutade@yahoo.com (T.Tadele), alemutamiso@yahoo.com (A. Tamiso), tafetade@yahoo.com (T. Tadele)

\section{To cite this article:}

Tariku Tadele, Alemu Tamiso, Tafese Tadele. Incidences and Predictors of HIV Positivity among Infants who Born from HIV Positive Mother who Have Follow Up at Two Hospitals of Southern Ethiopia, 2014. Science Journal of Public Health.

Vol. 2, No. 5, 2014, pp. 431-439. doi: 10.11648/j.sjph.20140205.19

\begin{abstract}
Introduction: Despite a dramatic progress in improvements regarding infant mortality due to HIV/AIDS in the past decades, HIV/AIDS is causing a devastating impact on the world's children. Mother-to-child transmission (MTCT) is by far the largest source of HIV infection in children under the age of 15 , with $90 \%$ of the cases infected during pregnancy, birth, and major share is after birth.Therefore, this study follows infants after the first test (Deoxyribonucleic acid (DNA) Polymerase Chain Reaction (PCR) testing) is negative, until the occurrences of HIV positivity and predictors associated with it in the two hospital of Southern Ethiopia. Objective: The aim was to assess the incidence, Survival experiences and predictors of HIV positivity among infants born from HIV positive mothers in two hospital of Southern Ethiopia, 2014. Methodology: Institution-Based Retrospective follow up study was conducted at Hawassa Referral and Yirgalem General Hospital from September 2000 to August 2005 E.C. Data was collected by trained BSc nurses from medical registration book of 485 exposed infants. It was entered in to EPI Info 3.5.1 and exported to SPSS version 20 for further analysis. Both Bivariate and Multiple variable Cox regression analysis were conducted to identify predictors. P-value $<0.05$ was considered as level of significance. Results: Among 457 participants included under analysis contributed for 4249.4 Person Months of follow up. Cumulative and overall incidence rate of HIV positivity among infants were 19(4.16\%) and 4.47/1000 PM (95\% CI: 4.02-4.92) respectively. Mean HIV free survival time difference between Exclusive breast feeding 20.2 (95\% CI, 19.6, 20.8)), mixed feeding (17.5 (95\% CI, 15.5-19.5)) and exclusive formula feeding 16.8 (95\% CI, 16.3, 18.4) were significant. Mixed breast feeding (AHR: 8.23(1.98, 34.2)), ARV prophylaxis (yes) (AHR: 0.19(95\% CI, 0.040.89)), Maternal HAART (AHR: 0.16(95\% CI, .041, 0.59) and SdNVP + AZT+ 3TC (AHR: 0.113(95\% CI: 0.02, 0.61)) intervention were independent predictors of HIV positivity among exposed infants. Conclusion: The risk of mother to child transmission of HIV is high for HIV exposed infants. All mothers should start PMTCT intervention as a guideline and it is better to discourage mixed infant feeding to prevent the upcoming infants from the HIV infection.
\end{abstract}

Keywords: HIV Positivity, Incidence Rate, Cox regression Model, Ethiopia

\section{Introduction}

Despite a dramatic progress in improvements regarding infant mortality due to HIV/AIDS in the past decades, HIV/AIDS is causing a devastating impact on the world's children(1). Globally, over 1,500 newborn babies are infected every day and over $90 \%$ of newly infected children are babies born to HIV-positive women, who acquire the virus at birth or through their mother's breast milk(2).

The rate of pediatric HIV infections in sub- Saharan 
Africa remains unacceptably high, with over 1,000 newborns infected with HIV per day (3). Pediatric HIV is a large contributor to the excessive infant and child mortality rates in sub-Saharan Africa. Thus the life expectancy of HIV-positive infants is extremely short. One-third of HIVpositive infants are estimated to die before their first birthday and over one-half will die by their second birthday(4).

Ethiopia is one of the country's most severely hit by the epidemic. According to Ethiopian demographic and health survey 2011, the national adult HIV prevalence is reported to be $1.5 \%$ (4.2\% in urban and $0.6 \%$ in rural areas)(5). Highest prevalence occurs in the 15-24 age groups and prevalence is higher among females $(1.9 \%)$ than males $(1.0 \%)$ in both urban and rural areas. Prevalence appears to have leveled off in urban areas but continues to rise in rural areas, where $85 \%$ of the population lives(6). Number of new HIV infections among children in Ethiopia at 2009 was 18,900 and this figures decrease to 13,000 in 2011(2).

Mother-to-child transmission (i.e., vertical transmission) of HIV is almost completely preventable through a set of interventions referred to as prevention of mother-to-child Transmission (PMTCT)(7). PMTCT begins during antenatal care (ANC) when the woman is tested for HIV and receives the result that she is HIV positive. the recommendation in sub-Saharan Africa is for the woman to then take medication throughout pregnancy, during labor, and the postnatal period while exclusively breastfeeding

Without treatment, approximately $25 \%-50 \%$ of HIVpositive mothers will transmit the virus to their newborns during pregnancy, childbirth, or breastfeeding (8). Some of the defined risk factors for MTCT include prenatal maternal factors such as high viral load, low CD4 cell count, and advanced clinical stage; obstetric factors such as prolonged rupture of membranes and invasive obstetrical procedures; and postnatal factors such as breastfeeding itself and breast conditions such as mastitis(7)

Ethiopia has adopted the World Health Organization (WHO) four pronged PMTCT strategy as a key entry point to HIV care for women, men and families. These are: primary prevention of HIV infection, prevention of unintended pregnancies among HIV-infected women, prevention of HIV transmission from HIV-infected women to their offspring, provision of care and support to women infected with HIV, their infants and families(9). The country has also developed an accelerated national PMTCT plan (2011-2015) and has adopted WHO 2010 Guidelines option ARegimen for prophylaxis(10). Despite marked progress to achieve zero MTCT by year 2015, the magnitude of MTCT still remains high in the country which is around $30 \%(4)$.

Except few research which study about HIV positivity among infants, in Ethiopia there is no research which used to study after the first test DNA polymerase chain reaction test is negative. So the aim of the study was to follow infants after the first test (DNA PCR) is negative, until the occurrences of HIV positivity and risk factors associated with it in the two hospital of Southern Ethiopia, 2014.

\section{Methodology}

\subsection{Study Area and Period}

The study was conducted in Hawassa University referral and Yirgalem general Hospital. Hawassa Referral hospital is located in Hawassa, the capital City of southern Nations, Nationalities and peoples Regional State. The city is located in the rift valley $275 \mathrm{Km}$ south of Addis Ababa. The hospital provides generalized and specialized services to more than 5 million people. Yirgalem general hospital is located $41 \mathrm{~km}$ south of Hawassa in sidama zone. It provides health services to more than 5 million populations. It is one of the oldest hospitals in Ethiopia. Apart from the other services, both of the hospitals are providing HIV chronic care including PMTCT and pediatric HIV follow up care since 1999 E.C.

\subsection{Study Design}

Institution-Based Retrospective follow up study was conducted among infants who had follow up at the pediatric HIV follow up clinic.

\subsection{Source Population}

All HIV exposed infants enrolled in to pediatric ART clinic for follow up in Hawassa Referral and Yirgalem general Hospital since their establishment.

\subsection{Study Population}

All HIV exposed infants who had follow up in Hawassa referral and Yirgalem general hospital from 2000 to 2005 E.C.

\subsection{Inclusion Criteria}

All HIV exposed infants who have DNA PCR at the age of enrollment below 18 months or rapid antibody test result during follow up after 6weeks of cessation of breast feeding.

\subsection{Exclusion criteria}

Infants enrolled in to the chronic cares that have no or incomplete chart or DNA PCR positive result during enrollment.

\section{Sample Size Determinations}

The required sample size was determined using Statecalc program of the EPI INFO version 7.1.0 statistical package with 5\% desired precision, $95 \%$ confidence level, $30 \%$ (Estimated risk of MTCT inEthiopia, UNAIDS 2012), and total population of 634(total infants with complete charts enrolled in to the two hospitals) yield 214 and twenty percent of loss to follow up, it give 257 study participants. $(257 \times 30 \%=77$ exposed). But to check whether or not sample size was sufficient to asses' determinants; Sample size was calculated for the second specific objectives. But 
sample size calculated first specific objective was 257 study participants was greater than calculated sample size for second specific objectives, so total 257 sample sizes was selected. At the end, in order to maximize the power of the study, all the infants (485) in both hospitals who fulfills inclusion criteria was included under the study

\subsection{Definition of Terms}

Abnormal breast conditions: any breast conditions like mastitis, ulcer, cracks or fissures.

Abnormal findings suggestive of HIV Infection: presence of persistent generalized lymphadenopathy, persistent diarrhea, pneumonia, persistent fever, etc

ARV prophylaxis: Short term use of ARV drugs in the mother and/or infant to reduce MTCT.

ART (Antiretroviral Therapy): is the use of 3 or more ARVs drugs simultaneously to treat HIV infection. ART is a life-long treatment for the mother and can also significantly reduce MTCT.

CPT adherence: is classified based on the number of missed doses per month (Good $\leq 3$ doses, Fair $=5-8$ and poor $\geq 9$ doses).

HIV positivity: itis based on a positive Antibody test done after 6 weeks of cessation of breast feeding or DNA PCR test after from 6 weeks to 18 months of age.

Incidence density: ID means number of HIV exposed infants with Positive rapid diagnostics test per1000 Person months of at risk of follow-up period. The risk period begins at the time of enrollment in to the HIV care and ends when HIV Antibody test was done and after become positive or transfer out, loss, drop, stop or end of the study.

Follow-Up of Infants Born to HIV-Positive Mothersin southern Ethiopia

According to the PMTCT 2011 guideline follow-up schedule is at 6 hours, 6 days, 6 weeks, 10 weeks, 14 weeks, and then monthly until 6 months, and thereafter every 3 months until 18 months if infant is asymptomatic. Cotrimoxazole prophylaxis is started at 4-6 weeks old and Deoxyribonucleic acid Polymerase Chain Reaction (DNAPCR) testing at 6 weeks or as early as possible ( for infants below age of 18 months) thereafter if not possible at 6 weeks and if on breast feeding Rapid antibody test is done after 6 weeks of cessation of breast feeding. The child will be referred for HIV/ART care clinic if child has a positive virological test or suspected of having symptomatic HIV or displays any severe classifications possibly due to HIV or has positive antibody test under 18 months and 2 or more of the following: oral thrush, severe pneumonia or severe sepsis.

\subsection{Data Collection}

Data was abstracted within 3 weeks from medical record and ART follow-up form for socio demographic (age, sex, residence), infant related health (mode of delivery, infant feeding, CPT prophylaxis, Immunization, Birth weight, place of delivery) and maternal characteristics (maternal breast status, HAART status, PMTCT follow up) by usingthreeBScnursesas data collector. The principal investigators were act as supervisor. Throughout the country the same medical chart and electronic ART followup form are used which was recommended by Federal Ministry of Health of Ethiopia. Simple and structured data collection questioner which is developed based on original medical and ART charts was used to facilitate reliable data abstraction.

\subsection{Data Quality Assurance}

In order to maintain the quality of data, BSc nurses who are trained on pediatric ART and working at ART clinic were selected for data collection. Training was given before data collection period for one dayabout objective of the study, variables on the questionnaire, medical record and follow-up form and how to abstract data for this study purpose. During data abstraction there was close supervision by principal investigators. Completed questionnaire was checked for completeness of information and any gap identified was immediately communicated to the data collectors.

\subsection{Data Management and Analysis}

Data was coded manually, entered and cleaned using EPI-INFO version 3.5.1 and exported to SPSS version 20 for descriptive and inferential analyses. STATA version 11.1 is used to check the proportional hazard assumptions. Frequencies and cross tabulations wereused to check for missed values and variables. HIV free survival time was estimated for different feeding options and Maternal PMTCT intervention type by using Kaplan- Meir curve. Log rank test was used in order to test whether the observed difference is significant or not. Multiple variables Cox regression analysis was conducted between HIV positivity of infants and maternal PMTCT intervention typeand feeding option adjusted for all possible confounding effect of other variable associated with HIV positivity.

$\mathrm{P}$-value $<0.05$ was considered as statistically significant. For Incidence Density ratio (IDR), Crude and Adjusted Hazard Ratio, survival probability, 95\% confidence interval and p-value was used to present the result of independent prediction of HIV positivity.

\subsection{Ethical Clearance}

Ethical clearance was obtained from Institutional Review office of Hawassa University, College of medicine and Health Science. Permission letter was obtained from Regional health Bureau to the Hawassa and Yirgalem hospital administration. As it is a retrospective study using secondary data, obtaining informed consent from individual patients was not possible. But, Confidentiality of the information extracted from charts was maintained by not recording their names from charts and keeping the extracted data from charts anonymous. 


\section{Result}

\subsection{Socio Demographic Characteristics of Infants}

From September 2000up to August 2005 E.C, a total of 634 exposed infants enrolled in to pediatric ART clinic of the two hospitals. Of them, 485 patients who fulfill inclusion criteria were included under the study. Among four hundred eight five infants $28(5.4 \%$ ) who already developed the outcome at enrolments were also excluded from the study. Two variables (birth weight and age at cessation of breast feeding) were missed in large and excluded from the analysis.

The mean and standard deviation (SD) of age at enrollments was $2.78( \pm 3.05)$ months. From the total participants $236(51.6 \%)$ were male and 338(74.0\%) were residents at urban area.

Majority $342(74.8 \%)$ of infants were born at health institution and $66.1 \%$ of infants took follow up at Hawassa referral hospital.

Baseline characteristics of patients by their outcome status are summarized in the table below.

Table 1. Socio-demographic characteristics of exposed infants by outcome status, southern Ethiopia, 2000 - 2005 E.C.

\begin{tabular}{llll}
\hline Characteristics & Censored \# & $\begin{array}{l}\text { HIV } \\
\text { Positivity }\end{array}$ & Frequency (\%) \\
\hline $\begin{array}{l}\text { Sex } \\
\text { Male }\end{array}$ & $229(97.0)$ & $7(3.0)$ & $236(51.6)$ \\
$\begin{array}{l}\text { Female } \\
\text { Name of hospital }\end{array}$ & $209(94.6)$ & $12(5.4)$ & $221(45.3)$ \\
Hawassa referral & $289(95.7)$ & $13(4.3)$ & $302(66.1)$ \\
Yirgalem regional & $149(96.1)$ & $6(3.9)$ & $155(35.9)$ \\
$\begin{array}{l}\text { Place of delivery } \\
\text { Institutional }\end{array}$ & $328(95.9)$ & $14(4.1)$ & $342(74.8)$ \\
$\begin{array}{l}\text { Home } \\
\text { Residence }\end{array}$ & $110(95.7)$ & $5(4.3)$ & $115(25.2)$ \\
Urban & $325(96.2)$ & $13(3.8)$ & $365(79.9)$ \\
Rural & $113(95.0)$ & $6(5.0)$ & $119(20.1)$ \\
$\begin{array}{l}\text { Age at enrolments } \\
>6 \text { weeks }\end{array}$ & $391(96.1)$ & $16(3.9)$ & $407(89.1)$ \\
$\leq 6$ weeks & $47(94.0)$ & $3(6.0)$ & $50(10.9)$ \\
Total & $438(96.8)$ & $19(4.2)$ & $457(100)$ \\
\hline
\end{tabular}

\subsection{Baseline and Infants Follow Up Characteristics}

The total 457 participants contribute for 4249.38 months of follow up. Median time of follow up was 9.1 months, (SD, 4.91). During follow up there were 19 disease cases, 121 lost to follow up/death, 79 on follow up, 13 unrecorded, and 225 infants were discharged. Table 2, 3and figures 1 and 2 below summarize socio demographic, baseline and follow up characteristics by the HIV positivity among infants.

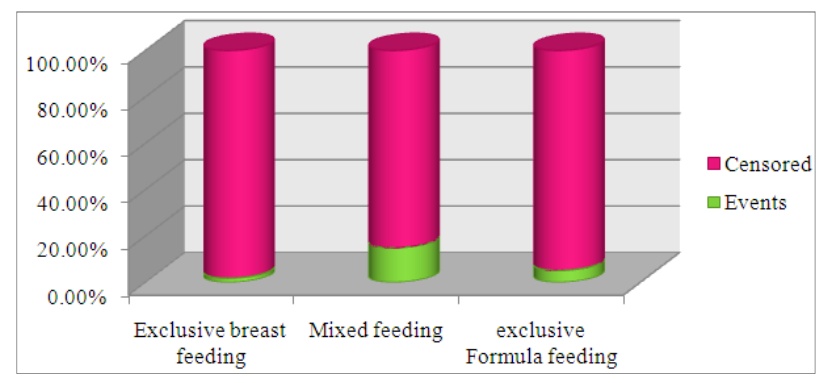

Figure 1. Different feeding option by outcome status in exposed infants, South Ethiopia, 2000 - 2005 E.C.

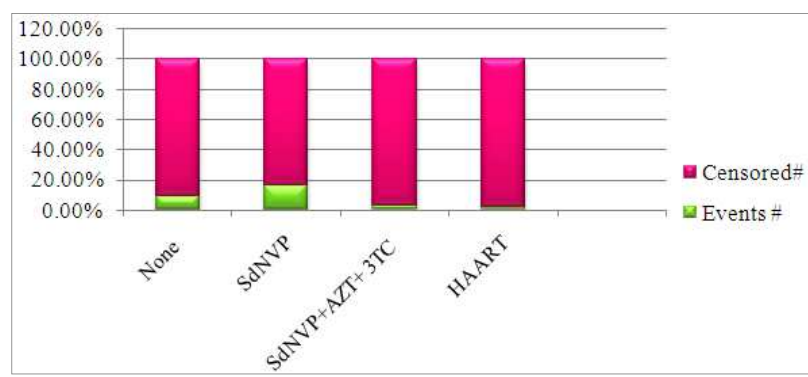

Foot note: SdNVP (single dose Nevirapine), AZT (Zidovudine), 3TC (Lamivudine), HAART (Highly Active Antiretroviral therapy)

Figure 2. Maternal PMTCT intervention type by outcome status at southern Ethiopia, 2000 - 2005 E.C.

Table 2. Incidence rate of HIV infection grouped by Socio-demographic characteristics, southern Ethiopia, 2000 - 2005 E.C

\begin{tabular}{|c|c|c|c|c|}
\hline Characteristics & Number & PM & HIV positivity & IDR (HIV Positive/PM Per 1000 months ) \\
\hline \multicolumn{5}{|l|}{ Sex } \\
\hline Male & 236 & 2251.24 & 7 & 3.12 \\
\hline Female & 221 & 1998.14 & 12 & 6.01 \\
\hline \multicolumn{5}{|l|}{ Age at enrolments } \\
\hline$\leq 6$ weeks & 50 & 488.2 & 3 & 6.1 \\
\hline$>6$ weeks & 407 & 3761.2 & 16 & 4.3 \\
\hline \multicolumn{5}{|l|}{ Residences } \\
\hline Urban & 338 & 3113.3 & 13 & 4.2 \\
\hline Rural & 119 & 1136.1 & 6 & 5.3 \\
\hline \multicolumn{5}{|l|}{ Place of delivery } \\
\hline Institutional & 342 & 3220.2 & 14 & 4.34 \\
\hline Home & 115 & 1029.2 & 5 & 4.85 \\
\hline \multicolumn{5}{|c|}{ Hospital where infants took follow up } \\
\hline Hawassa & 302 & 2550 & 13 & 5.1 \\
\hline Yirgalem & 155 & 1699 & 6 & 3.53 \\
\hline Total & 457 & 4249.38 & 19 & 4.47 \\
\hline
\end{tabular}


Table 3. Incidence rate of HIV infection grouped by baseline and follow up characteristics of infants and maternal health characteristics, Southern Ethiopia, 2000 - 2005

\begin{tabular}{|c|c|c|c|c|}
\hline Characteristics & Number & $\mathbf{P M}$ & Event & IDR (HIV Positive/PM Per 1000 months \\
\hline \multicolumn{5}{|l|}{ Infants ARV prophylaxis } \\
\hline Received & 286 & 1504.8 & 9 & 4.0 \\
\hline Not received and others & 171 & 2744.6 & 10 & 5.31 \\
\hline \multicolumn{5}{|l|}{ Adherence to CPT } \\
\hline Good & 270 & 2648.8 & 12 & 4.5 \\
\hline Fair \& poor & 187 & 1584.4 & 7 & 4.42 \\
\hline \multicolumn{5}{|l|}{ Immunization status } \\
\hline Complete & 306 & 2798.3 & 7 & 2.14 \\
\hline Unimmunized & 21 & 434.3 & 2 & 6.91 \\
\hline Incomplete & 130 & 1016.75 & 10 & 9.84 \\
\hline \multicolumn{5}{|c|}{ Type of infants feeding option } \\
\hline Exclusive breast feeding & 331 & 2999.4 & 6 & 0.2 \\
\hline Mixed feeding & 67 & 725.5 & 10 & 13.8 \\
\hline Formula feeding & 59 & 524.3 & 3 & 5.74 \\
\hline \multicolumn{5}{|c|}{ Abnormal finding suggestive of HIV } \\
\hline Yes & 414 & 406.9 & 4 & 1.04 \\
\hline No & 43 & 3842 & 15 & 36.8 \\
\hline \multicolumn{5}{|c|}{ Maternal health characteristics } \\
\hline Died and others & 51 & 490.4 & 3 & 6.1 \\
\hline Alive & 406 & 3758.9 & 16 & 4.2 \\
\hline \multicolumn{5}{|c|}{ Maternal PMTCT intervention } \\
\hline Yes & 372 & 778.6 & 12 & 3.17 \\
\hline No & 85 & 3470.7 & 7 & 10.24 \\
\hline \multicolumn{5}{|l|}{ Type of maternal PMTCT } \\
\hline None & 86 & 781.3 & 8 & 10.2 \\
\hline SdNVP & 25 & 173.71 & 4 & 23.03 \\
\hline $\mathrm{SdNVP}+\mathrm{AZT}+3 \mathrm{TC}$ & 119 & 1196.69 & 3 & 2.51 \\
\hline HAART & 227 & 2097.5 & 4 & 0.48 \\
\hline \multicolumn{5}{|l|}{ Maternal breast condition } \\
\hline Normal & 444 & 4109.5 & 16 & 3.89 \\
\hline Diseased & 13 & 139.83 & 3 & 2.14 \\
\hline Total & 457 & 4249.2 & 19 & 4.47 \\
\hline
\end{tabular}

Foot note: SdNVP (single dose Nevirapine), AZT (Zidovudine), 3TC (Lamivudine), HAART (Highly Active Antiretroviral therapy)

\subsection{Incidence Rate of HIV Positivity}

The overall incidence rate of HIV infection was 4.47/1000 PM (95\% CI: 4.02-4.92). Total number of positive cases is $19(4.16 \%)$. The tables below summarize incidence rate of HIV infection according to different characteristics.

\subsection{Survival Experience for Different Category}

The overall cumulative probability of HIV free survival for the total subject at the end of the study was 0.96 and mean for survival time was 20.054 (95\% CI, 19.43 - 20.68) months. The cumulative HIV free probability and mean for survival time was 0.98 and 20.2 (95\% CI, 19.62-20.78) months for exclusive breast feeding option and 0.85 and 17.50 (95\% CI, 15.46-19.54)months for mixed feeding and 0.95 and $16.83(95 \% \mathrm{CI}, 16.30-18.36)$ months for formula feeding option. Log rank test for infants feeding option strata was significant ( $p$-value $<0.001$ ).

The overall cumulative probability of HIV free survival and mean for survival time for PMTCT Intervention type was 0.91 and $18.8(95 \% \mathrm{CI}=17.3,20.3)$ months for PMTCT non utilized mother, 0.84 and 13.1 (95\% CI $=1.45$, 10.34) months for SdNVP utilized mother, 0.98 and $18.5(95 \% \mathrm{CI}=17.9,19.2)$ for $\mathrm{SdNVP}+\mathrm{AZT}+3 \mathrm{TC}$ and 0.98 and $20.1(95 \% \mathrm{CI}=19.3,20.9)$ for HAART with the logrank test ( $\mathrm{p}$-value of 0.001). Kaplan- Meier survival curve were presented below. 


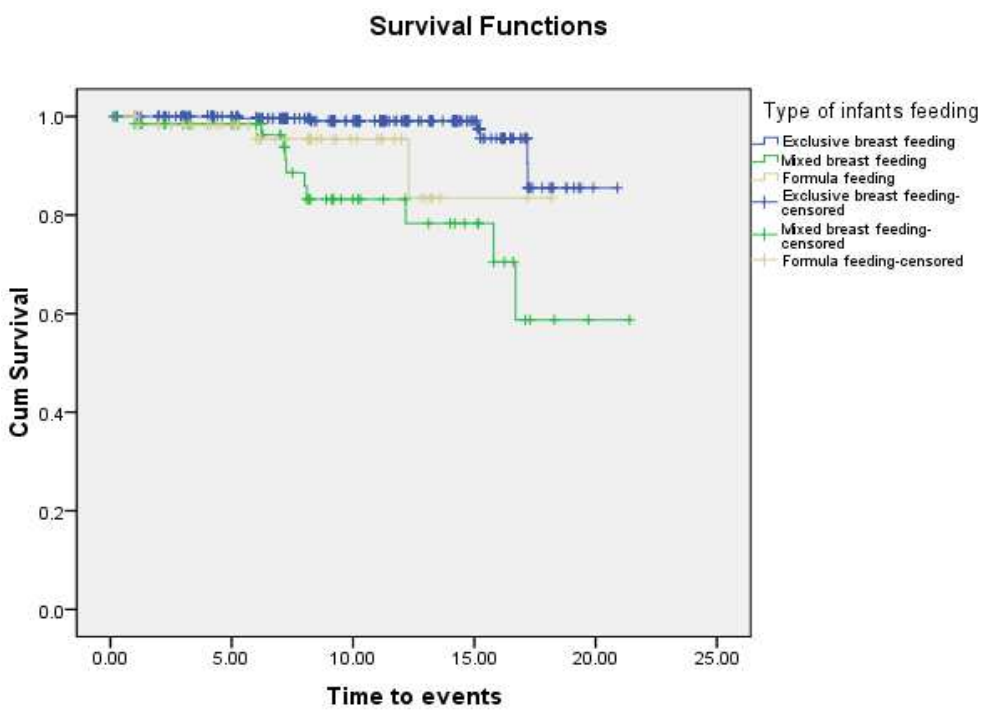

Figure 3. HIV free survival functions of exposed infants feeding option (Log Rank test: P-Value <0.001), Southern Ethiopia, 2000 - 2005E.C.

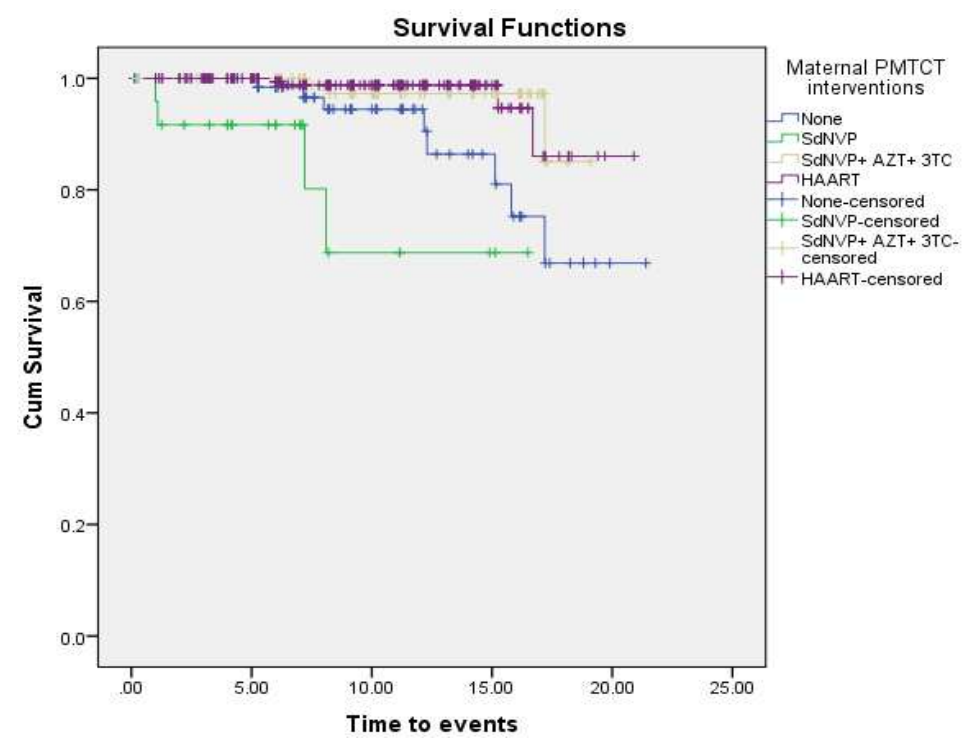

Figure 4. HIV free survival functions for exposed infants on Maternal PMTCT intervention type, (Log Rank test: P-Value <0.001), southern Ethiopia, $2000-2005 E . C$.

\subsection{Predictors of HIV Positivity}

To identify the predictors, nine variables which include: Follow up hospital, Infant ARV prophylaxis, sex of infant, maternal PMTCT intervention, abnormal finding suggestive of HIV, different feeding option, type of PMTCT intervention, immunization status and maternal breast condition which had association with HIV free survival time at $\mathrm{P}$-Value $<0.02$ on bivariate analysis among exposed infants were taken to multiple Cox regressional analysis.

Before multiple Cox regression analysis, assumption of proportional hazard ratio was checked by using graphical and statistical method. Finding from graphical method for Different feeding option looks like parallel throughout, but one strata pass other at the beginning and finding from global goodness-of-fit test (Schoenfeld's method) for different feeding option was proportional overtime (pvalue $=0.352$ ). This is also true in case of infants immunization status for which the graphical method violates the assumption but the finding from global goodness-of-fit test (Schoenfeld's method) for immunization status was proportional overtime ( $p$ value=0.882). On multiple variables Cox regression analysis: different feeding option, infant ARV prophylaxis, strata of PMTCT Intervention, and abnormal finding suggestive of HIV positivity were statistically significant. Table six below will summarize the finding.

Maternal PMTCT intervention (yes, no), maternal breast condition and immunization status were significant at bivariate Cox regression analysis but insignificants at multivariable Cox regression analysis. (Table 4 four summarizes the results) 
Table 4. Predictors of HIV free survival time in Cox regression, southern Ethiopia, 2000 - 2005 E.C

\begin{tabular}{|c|c|c|c|c|c|}
\hline Characteristics & Number & $\mathbf{P M}$ & Event & CHR (95\% CI) & AHR (95\% CI) \\
\hline \multicolumn{6}{|c|}{ Hospital were infants took follow up } \\
\hline Hawassa & 302 & 2550 & 13 & $1.93(0.72,5.18)$ & $* *$ \\
\hline Yirgalem & 155 & 1699 & 6 & 1 & \\
\hline \multicolumn{6}{|l|}{ Sex of exposed infants } \\
\hline Male & 236 & 2251.2 & 7 & 1 & $* *$ \\
\hline Female & 221 & 1998.1 & 12 & $0.51(.20,1.29)$ & \\
\hline \multicolumn{6}{|l|}{ Infants ARV prophylaxis } \\
\hline Received & 286 & 1504.8 & 9 & $0.45(0.18-1.102)$ & $0.188(0.04-0.89) *$ \\
\hline Not received & 171 & 2744.6 & 10 & 1 & 1 \\
\hline \multicolumn{6}{|l|}{ Immunization status } \\
\hline Complete for age & 306 & 2798.3 & 7 & $0.25(0.09,0.67)$ & ** \\
\hline Incomplete for age & 21 & 434.3 & 2 & $1.36(0.29,6.62)$ & \\
\hline Unimmunized & 130 & 1016.7 & 10 & 1 & \\
\hline \multicolumn{6}{|l|}{ Maternal PMTCT status } \\
\hline Received & 372 & 778.6 & 7 & 1 & \\
\hline Not received & 85 & 3470.7 & 12 & $2.37(0.93,6.02)$ & $* *$ \\
\hline \multicolumn{6}{|c|}{ Abnormal finding suggestive of HIV } \\
\hline Yes & 43 & 406.9 & 15 & 1 & $39.02(10.9-138.8) *$ \\
\hline No & 414 & 3842 & 4 & $39.5(12.9,120.6)$ & 1 \\
\hline \multicolumn{6}{|c|}{ Type of PMTCT intervention } \\
\hline None & 86 & 781.3 & 8 & 1 & 1 \\
\hline SdNVP & 25 & 173.71 & 4 & $4.16(1.19-14.6)$ & $2.86(0.69,11.9)$ \\
\hline $\mathrm{SdNVP}+\mathrm{AZT}+3 \mathrm{TC}$ & 119 & 1196.7 & 3 & $0.25(.07,0.93)$ & $0.113(0.02,0.61) *$ \\
\hline HAART & 227 & 2097.5 & 4 & $0.23(0.07,0.78)$ & $0.166(.041,0.59) *$ \\
\hline \multicolumn{6}{|l|}{ Infants feeding options } \\
\hline Exclusive breast feeding & 331 & 2999.4 & 6 & 1 & 1 \\
\hline Mixed breast feeding & 67 & 725.5 & 10 & $9.06(3.29,24.9)$ & $8.23(1.98,34.2) *$ \\
\hline Formula feeding & 59 & 524.3 & 3 & $4.55(1.13,18.4)$ & $4.86(0.99,23.7)$ \\
\hline \multicolumn{6}{|l|}{ Maternal breast condition } \\
\hline Normal & 444 & 4109.5 & 16 & 1 & \\
\hline Diseased & 13 & 139.83 & 3 & $7.34(2.09-25.7)$ & $* *$ \\
\hline
\end{tabular}

SdNVP (single dose Nevirapine), AZT (Zidovudine), 3TC (Lamivudine), HAART (Highly Active Antiretroviral therapy)

* Significant at P-Value $<0.05$.

** Non Significant from the multivariate Cox Regression (Backward LR Method)

\section{Discussion}

Despite the scientifically proven way of prevention of mother to child transmission of HIV, still more than ninety percent of pediatric HIV infections in resource limited countries occur vertically during pregnancy, delivery or breastfeeding period. In this retrospective follow up study the overall and stratified incidence density rate of HIV infection among exposed infants and cumulative survival probability between infants feeding option and between maternal PMTCT intervention typesgrouped into four were assessed.

The result of this study states the overall incidence rate of HIV infection was 4.47/1000 PM and cumulative HIV positivity at the end of the study was $4.2 \%(3.97,4.42)$. It was higher than study in western country $(2 \%)$, but lower than the study in India (5.9\%) as well study in SouthAfrica $(6.9 \%)(11-14)$. This may be due to universal access to PMTCT interventions in developed countries and limited access in resource limited countries as a result of lack of access, lack of awareness, quality of service and others. The study in north Ethiopia also showed higher HIV positivity among HIV exposed infants. These could be explained by the difference of the method, where this study only used final HIV test done after 3 months of cessation of breast feeding to diagnose HIV to estimate the incidence rate and other mentioned study used HIV test at 6 week and at the end of follow up to calculate the incidences.

In the analysis of mean for survival time among feeding option and grouped maternal PMTCT intervention type,there wasa statistically significant difference. These results are consistent with the study done in Ethiopia and comparable with previous prospective and randomized control trials from South Africa $(18,19)$.

From result of ordinary Cox regression analysis infants feeding option, infants ARV prophylaxis, maternal PMTCT intervention and abnormal finding suggestive of HIV infection becomes significantly associated with risk of HIV infection on the cohort of exposed infants.

An infant with mixed feeding option was 8.23 times at higher risk of developing HIV infection than infants on exclusive breast feeding counterparts. But there are no significant differences between exclusive replacements feeding and exclusive breast feeding. The finding was consistent with thestudy done in South Africa that mixed feeding on infants increase the hazard of HIV infection but there is no statistically significant difference between exclusive breast feeding and exclusive formula feeding. 
Study done Tamil nadu district in India showed also exclusive breast feeding can decrease the risk of HIV positivity, the finding also supported by the retrospective follow up study in north west Ethiopia, that mixed feeding can increase risk of HIV positivity among infants(13-15).

Infants ARV prophylaxis at birth was not significant at bivariate analysis but significant at multivariate Cox regressional analysis, thus compared to infants who not took antiretroviral prophylaxis at birth, infants who took ARV prophylaxis at birth was $81.2 \%$ less risk of developing HIV positivity, this was consistent with the study done in south Africa and cohort study done in Abidjan, Nigeria $(16,17)$. the finding also supported in the retrospective study done in Addis Ababa Ethiopia(18). This result is in line with the widely accepted fact, as providing ARV for infants will decrease the risk of infection.

Besides infant's prophylaxis, maternal PMTCT intervention type was significantly associated with HIV positivity among exposed infants. Accordingly infants who are born from mother who took HAART and SdNVP+AZT+3TC were $84.4 \%$ and $88.7 \%$ less risk of developing HIV positivity respectively, than infants who bornfrom mother who didn't took PMTCT intervention. The finding was consistent with the study done in South Africa (16); and Ethiopia(15).

Finally infant with abnormal finding suggestive of HIV was 39.02 times higher risk of developing HIV infection than infants with no sign. This might be due to Immunosuppressionleading to occurrence of opportunist infection.

Based on the result of global goodness-of-fit test time dependent Cox regression was performed for immunization status but the interaction variable becomes statistically nonsignificant. As a result the ordinary multivariable Cox regression analysis was done yielding insignificant result. In addition to this, maternal PMTCT intervention (yes, no) and maternal breast condition were significant at bivariate Cox regression analysis but insignificants at multivariable Cox regression analysis. This might be due to incompleteness of data, sample size and exclusion of HIV positive infants during the start of the study.

\section{Conclusion}

There is a high risk of mother to child transmission of HIV infection among infants born form HIV positive mothers who had follow up at two hospital of Ethiopia during the study period. The risk was higher among infants who had mixed feeding, abnormal findings suggestive of HIV and lower among infants who took ARV prophylaxis and whose mothers took antiretroviral therapy. So All mothers should start PMTCT intervention as a guideline to prevent their upcoming infants from the HIV infection and it is better to strengthen the status of PMTCT at health institution so as to decrease HIV infection among infants who born from HIV infected mother. In addition, institutional delivery is encouraged and mixed feeding is discouraged among HIV infected women. Prospective cohort study should be conducted by including all possible confounding variables not included under this study and to avoid lost to follow up problem.

\section{Acknowledgements}

The authors are very grateful to Hawassa referral hospital and Yirgalem general hospital for its administrative and technical assistance. The school of public health, college of medicine and health science, Hawassa University deserves acknowledgement for as financial support for this, study.

Authors' contributions: ${ }^{1} \mathrm{TT}$ was the primary researcher, conceived the study, designed, participatedin data collection, conducted data analysis, drafted and finalized themanuscript for publication. ${ }^{2}$ ATwas assisted in data collection andreviewed the initial and final drafts of the manuscriptand ${ }^{2}$ TTinterpreted the results, and reviewed the initial and final drafts of themanuscript.All authors read and approved the final manuscript.

\section{List of Abbreviation}

AHR: adjusted hazard rate, CPT: Cotrimoxazole prophylaxis therapy, HAART: Highly Active Anti-retroviral Therapy, IDR: Incidence Density ratio,KM: Kaplan Meir, MOH: Ministry of Health, PM: Person month,PMTCT: Prevention of Mother-To-Child Transmission of HIV,YGH: Yirgalem general hospital,

\section{Competing Interests}

The authors declare that there is no competing interest.

\section{References}

[1] AVERT. An international AIDS Charity. Avert.org Worldwide HIV/AIDS epidemic statistics, 2005, http://www.avert.org/worlstatinfo.htm.

[2] UNAIDS: UNAIDS Report on the global AIDS epidemic 2012. Geneva; 2012.

[3] M. Besser, Preventing Mother-to-Child HIV Transmission in Africa Using New Paradigms in Health Care Delivery: Mothers to Mothers, 2010.

[4] S. Lucas, "Causes of death in the HAART era," Current Opinion in Infectious Diseases, vol. 25, no. 1, pp. 36-41, 2012.

[5] CSA, ICF International: Ethiopia Demographic and Health Survey 2011, Addis Ababa, Ethiopia and Calverton, Maryland, USA: Central Statistical Agency and ICF International; 2012.

[6] FMoH: Guidelines for Prevention of Mother-to-Child Transmission of HIV in Ethiopia. Addis Ababa, Ethiopia: Federal HIV/AIDS Prevention and Control Office; 2007. 
[7] Michael A DD. Prevention of Mother-to-Child Transmission of HIV Infection. 2010.

[8] Chowdhury M, Ahmed A, Kalim N, Koblinsky M. Causes of maternal mortality decline in Matlab, Bangladesh. J Health Popul Nutr 2009;27(2):108-123.

[9] Prevention of Mother to Child Transmission (PMTCT). http://www.unicef.org/supply/index_42855.html.

[10] MOH. Guidelines For Prevention of Mother-to-Child Transmission of HIV In Ethiopia Addis Abeba: 2007.

[11] Thorne C, Patel D, Fiore S, Peckham C, Newell M-L: Mother-to-child transmission of HIV infection in the era of highly active antiretroviral therapy. Clin Infect Dis 2005, 40(3):458-465.

[12] MoFED, Growth and Transformation Plan-Annual Progress Report (GTP-APR 2010/11), Addis Ababa, (2010/11), Ethiopia.

[13] Coovadia HM, Rollins NC, Bland RM, Little K, Coutsoudis A, Bennish ML, et al. Mother-to-child transmission of HIV1 infection during exclusive breastfeeding in the first 6 months of life: an intervention cohort study. Lancet. 2007;369(9567):1107-16. Epub 2007/04/03.

[14] Read JS, Samuel NM, Srijayanth P, Dharmarajan S, Van
Hook HM, Jacob $M$, et al. Infants of human immunodeficiency virus type 1-infected women in rural south India: feeding patterns and risk of mother-to-child transmission. Pediatr Infect Dis J. 2010;29(1):14-7. Epub 2009/11/17.

[15] Koye Digisu ZB. Mother-to-child transmission of HIV and its predictors among HIV-exposed infants at aPMTCT clinic in northwest Ethiopia. BMC Public Health. 2011;13(398):6. Epub 2013.

[16] Hoffman RM, Black V, Technau K, van der Merwe KJ, Currier J, Coovadia A, et al. Effects of highly active antiretroviral therapy duration and regimen on risk for mother-to-child transmission of HIV in Johannesburg, South Africa. J Acquir Immune Defic Syndr. 2010;54(1):35-41. Epub 2010/03/11.

[17] Dabis F, Bequet L, Ekouevi DK, Viho I, Rouet F, Horo A, Sakarovitch C, Becquet R, Fassinou P, Dequae-Merchadou L, et al: Field efficacy of zidovudine, lamivudine and singledose nevirapine to prevent peripartum HIV transmission. AIDS 2005, 19(3):309-318.

[18] Mirkuzie AH, Hinderaker SG, Morkve O: Promising outcomes of a national programme for the prevention of mother-to-child HIV transmission in Addis Ababa: a retrospective study. BMC Health Serv Res 2010, 10:267. 